\title{
Long non-coding RNA expression profile in minor salivary gland of primary Sjögren's syndrome
}

\author{
Huan $\mathrm{Shi}^{\dagger}$, Ningning $\mathrm{Cao}^{\dagger}$, Yiping Pu, Lisong Xie, Lingyan Zheng ${ }^{*}$ and Chuangqi Yu ${ }^{*}$
}

\begin{abstract}
Background: To examine the roles of long noncoding RNAs (IncRNAs) in the regulation of primary Sjögren's syndrome (pSS) and reveal the expression profile of IncRNAs in labial salivary glands (LSGs) in pSS patients.

Method: The expression of 63,431 IncRNAs and 39,887 mRNAs were determined in the LSG of four pSS patients and four healthy controls using microarray experiments. Validation was performed in 30 pSS patients and 16 controls using real-time PCR. LncRNA-mRNA co-expression and gene-pathway networks were constructed using bioinformatics software.

Result: A total of 1243 IncRNAs (upregulated: 890, downregulated: 353) and 1457 mRNAs (upregulated: 1141, downregulated: 316 ) were differentially expressed in the LSGS of pSS patients (fold change $>2, P<0.05$ ). Eight of these IncRNAs were validated using real-time PCR. ENST00000420219.1 (3.13-fold), ENST00000455309.1 (2.51-fold), n336161 (2.45-fold), NR_002712 (2.41-fold), ENST00000546086.1 (1.94-fold), Lnc-UTS2D-1:1 (1.79-fold), n340599 (1.69-fold), and TCONS_12_00014794 (1.28-fold) were significantly upregulated in pSS. There were strong correlations between these IncRNAs and $\beta 2$ microglobulin, disease course, erythrocyte sedimentation rate (ESR), rheumatoid factor (RF), IgA, IgM, visual analogue scale (VAS) of parotid swelling and VAS of dry eyes. Computational analyses revealed that 28 of the differentially expressed (DE) mRNAs were associated with eight DE IncRNAs involved in chemokine signaling pathways, the nuclear factor-kappa B (NF-kB) signaling pathway, and tumor necrosis factor (TNF) signaling pathway.

Conclusions: Our study revealed the expression profile of IncRNAs in LSGs of pSS patients. Many novel IncRNA transcripts that play important roles in the pathogenesis of pSS were dysregulated in pSS. Therefore, this study will aid in the development of new diagnostic biomarkers and drug therapies.
\end{abstract}

Keywords: Long noncoding RNA, Sjögren's syndrome, Expression profile, Pathogenesis

\section{Background}

Primary Sjögren's syndrome (pSS) is an autoimmune disease that is characterized by the dysfunction of exocrine glands, primarily the salivary and lachrymal glands, which results in dry mouth and eyes. The extensive infiltration of sensitized lymphocytes into target glands leads to the primary pathological manifestations of pSS. The etiology of pSS not clear, but previous studies have suggested that hereditary, hormonal, and environmental

\footnotetext{
*Correspondence: zhenglingyan73@163.com; yca616@hotmail.com ${ }^{\dagger}$ Equal contributors

Department of Oral Surgery, Affiliated Shanghai Ninth People's Hospital, Shanghai Jiao Tong University School of Medicine, Shanghai Key Laboratory of Stomatology, Shanghai, China
}

factors play crucial roles in the onset and progression of pSS [1]. Activation of the innate/adaptive immune system is the first line of defense against infections and damaged tissues. However, aberrantly activated inflammatory processes underlie autoimmune disease. Persistent perturbations of these inflammatory pathways are detrimental to the host and eventually result in disease conditions, including rheumatoid arthritis (RA), cardiovascular disease, and cancer [2-4]. Therefore, inflammatory signaling pathways require strict regulation at the transcriptional and posttranscriptional levels. Recent studies have demonstrated that noncoding RNAs, especially microRNAs, play crucial roles in the regulation of 
inflammatory signaling pathways [5, 6]. The roles of long noncoding RNAs (lncRNAs) as novel regulators of these pathways have emerged in more recent years. LncRNAs are a newly discovered class of regulatory molecules that are not translated into proteins and consist of more than 200 nucleotides. LncRNAs strongly affect a variety of biological processes in cells and organ systems. Several studies revealed the strong involvement of lncRNAs in the regulation of the immune response, including several pathways of innate immunity $[7,8]$. LincRNA-Cox2 is one IncRNA that both negatively and positively regulates the expression of many important immune-related genes. This IncRNA mediates its repressive effects on interferonstimulated genes via interactions with hnRNP-A/B and A2/B1 [9, 10]. Recent studies have also identified THRIL as a key regulator of tumor necrosis factor alpha (TNF- $\alpha$ ) induction on Toll-like receptor (TLR) $1 / 2$ signaling in human THP1 macrophages. Knockdown of THRIL downregulated the production of TNF- $\alpha$ mRNA [11]. Several other lncRNAs, such as NEAT [12, 13], Inc-IL7R [14], PACER [15], lnc-DC [16], IL1 $\beta$-RBT46 [17] and AS-IL1 $\alpha$ [18], control the innate immune response, immune cell development, and adaptive immunity. These studies indicate the crucial role of IncRNAs in the normal immune system. However, it is important to identify whether lncRNA dysfunction is involved in the pathogenesis of autoimmune diseases. Increasing evidence suggests that the dysregulation of lncRNAs plays an important role in autoimmune diseases, such as systemic lupus erythematosus (SLE), RA, type I diabetes mellitus (T1DM), and multiple sclerosis (MS) [19-24]. The present study analyzed the lncRNA expression profiles in labial salivary glands (LSGs) of pSS patients using lncRNA microarray to investigate the potential roles of lncRNAs in the pathogenesis of pSS. The results provide a new direction for the diagnosis and therapy of pSS.

\section{Methods}

\section{Study subjects}

Thirty patients diagnosed with pSS and 16 control subjects were recruited from the Department of Oral Surgery, Shanghai Ninth People's Hospital, School of Medicine, Shanghai Jiao Tong University. All of the selected pSS patients fulfilled the American-European consensus group criteria for pSS. Specimens were collected during labial biopsy. No immunosuppressive treatment was administered to patients prior to diagnosis. Sixteen control subjects were diagnosed with labial gland mucocele and underwent mucocele excision. Normal labial glands adjacent to mucocele were collected during surgery. The absence of acute infections or systemic diseases was confirmed in all healthy donors. All specimens were immediately frozen in liquid nitrogen after resection and stored at $-80{ }^{\circ} \mathrm{C}$ until RNA extraction. The discovery cohort was composed of eight patients mixed with pSS patients and control subjects for the screening of 63,431 lncRNA transcripts. The independent validation cohort consisted of $30 \mathrm{pSS}$ patients and 16 normal samples. Table 1 summarizes the detailed demographic, clinical and laboratory characteristics of the $30 \mathrm{pSS}$ patients. All healthy donors were females aged 25-65 years old. Consent was obtained from each participant prior to sample collection. The Ethics Committee, Faculty of Medicine, Shanghai Jiao Tong University approved this study.

\section{Microarray}

Total RNA was extracted from eight samples (four pSS and four control subjects) using TRIzol reagent (Life

Table 1 The detailed demographic, clinical, and laboratory characteristics of 30 pSS patients

\begin{tabular}{|c|c|}
\hline Characteristic & pSS patient \\
\hline Sex, no. male/female & $0 / 30$ \\
\hline Age, mean $\pm S D$ years & $47.63 \pm 13.50$ \\
\hline Dry mouth, VAS. mean \pm SD & $6.57 \pm 2.12$ \\
\hline Dry eyes, VAS. mean \pm SD & $2.90 \pm 2.95$ \\
\hline Parotid swelling, VAS. mean \pm SD & $4.00 \pm 2.40$ \\
\hline Rose bengal score, no. +/- & $20 / 10$ \\
\hline Saxon test (g/2 min). mean $\pm S D$ & $1.44 \pm 0.62$ \\
\hline \multicolumn{2}{|l|}{ Grading of labial salivary gland biopsies, no. } \\
\hline Nonspecific chronic sialadenitis & 1 \\
\hline Grade 1 (<50 periductal lymphocytes) & 1 \\
\hline \multicolumn{2}{|l|}{ Grade 2 (>50 periductal lymphocytes) } \\
\hline Nonsegregated & 6 \\
\hline Segregated aggregates & 6 \\
\hline $\begin{array}{l}\text { Grade } 3 \text { ( }>50 \text { periductal lymphocytes, with GC-like } \\
\text { structures) }\end{array}$ & 16 \\
\hline Ro (SSA), no. +/- & $24 / 6$ \\
\hline La (SSB), no. +/- & $10 / 20$ \\
\hline Anti-centromere antibodies (ACA), no. +/- & $6 / 24$ \\
\hline $\mathrm{ESR}(\mathrm{mm} / \mathrm{hr}) \cdot$ mean $\pm \mathrm{SD}$ & $21.77 \pm 12.95$ \\
\hline $\mathrm{RF}(\mathrm{IU} / \mathrm{ml})$. mean $\pm \mathrm{SD}$ & $146.21 \pm 195.12$ \\
\hline $\operatorname{lgG}(\mathrm{g} / \mathrm{L})$. mean $\pm \mathrm{SD}$ & $19.12 \pm 4.10$ \\
\hline $\lg A(g / L) . ~ m e a n ~ \pm S D$ & $3.22 \pm 0.84$ \\
\hline $\operatorname{lgE}(\mathrm{IU} / \mathrm{ml})$. mean $\pm \mathrm{SD}$ & $78.54 \pm 68.92$ \\
\hline $\operatorname{lgM}(\mathrm{g} / \mathrm{L})$. mean $\pm S D$ & $1.73 \pm 0.74$ \\
\hline Course of disease (month). mean \pm SD & $35.53 \pm 26.04$ \\
\hline $\mathrm{CRP}(\mathrm{mg} / \mathrm{L})$. mean $\pm \mathrm{SD}$ & $4.46 \pm 2.15$ \\
\hline C3 (g/L). mean $\pm S D$ & $1.10 \pm 0.19$ \\
\hline C4 (g/L). mean $\pm S D$ & $0.26 \pm 0.07$ \\
\hline$\beta 2$ microglobulin(mg/L). mean $\pm S D$ & $2.80 \pm 0.88$ \\
\hline
\end{tabular}

pSS primary Sjögren's syndrome, SD standard deviation, VAS visual analogue scale, ESR erythrocyte sedimentation rate, $\mathrm{RF}$ rheumatoid factor, $1 \mathrm{~g}$ immunoglobulin, CRP C-reactive protein, C3 complement 3, C4 complement 4 
Technologies, Carlsbad, CA, USA). The RNeasy Mini Kit (Qiagen, GmBH, Hilden, Germany) was used to purify the total RNA according to the manufacturer's recommendation. Purified total RNA was quantified using a NanoDrop 1000 (Thermo Fisher Scientific, Waltham, MA, USA). The assessment of RNA integrity was determined using RNA LabChip ${ }^{\text {тs }}$ kits and an Agilent 2100 bioanalyzer (Agilent Technologies, Santa Clara, CA, USA). Only samples with 2100 RIN $\geq 7.0$ and $28 S / 18 S \geq 0.7$ were used.

Agilent SurePrint G3 microarray was used to investigate 63,431 lncRNAs and 39,887 mRNAs. Total RNA was amplified and labeled using a Low Input Quick Amp Labeling Kit, One-Color (Agilent Technologies, Santa Clara, CA, US). Labeled cRNA were purified using an RNeasy mini kit (Qiagen, GmBH, Hilden, Germany). The microarray hybridization was performed based on the manufacturer's standard protocols (Agilent Technologies, Santa Clara, CA, US). Slides were washed in staining dishes and scanned. Raw data were normalized using a Quantile algorithm in Gene Spring Software 11.0 (Agilent Technologies, Santa Clara, CA, US).

\section{LncRNA-mRNA co-expression network}

An IncRNA-mRNA co-expression network was built based on the normalized signal intensity of differentially expressed lncRNAs and mRNAs to explore the dysregulation of lncRNAs in pSS patients. A coexpression network of control subjects and pSS patients was established. Differentially expressed lncRNAs and mRNAs that met the criteria ( $P$ value $<0.05$, fold change $>2$ or $<0.5)$ were selected. Correlations between lncRNA and lncRNA, lncRNA and mRNA, mRNA and mRNA were investigated using Pearson's correlations. Only strong correlations $(P<0.001)$ were drawn in these renderings. The importance of a gene in this network is reflected by degree. A gene with a large degree indicated that it was at a central position in the network and it shared closer relationships with more genes. The last step investigated genes that exhibited different degrees in pSS patients and control subjects.

\section{Screening of differentially expressed genes for validation}

We screened differentially expressed (DE) lncRNAs for further validation using the following two approaches to validate the results of microarray experiments in an independent cohort and investigate the correlations between gene expression levels and clinical characteristics.

First, genes were evaluated based on the data revealed by microarray experiments using the following criteria: (a) the fold change of genes must be greater than fivefold compared to control subjects; (b) the signal value of the probes in each sample must be greater than seven; (c) the signal of the probes in each sample must be significantly different from the background signal; and (d) genes with
lncRNA-mRNA repeated sequences and without information in databases were excluded.

Second, the degree of differentially expressed genes between pSS patients and control subjects was compared based on the co-expression network results. The top 30 genes with high different degrees and the qualified requirements for signal values were selected.

\section{Real-time PCR}

Total RNA was extracted as described above. cDNA was synthesized from $0.5 \mu \mathrm{g}$ RNA using the iScript cDNA synthesis kit (Bio-Rad Laboratories, Hercules, CA, USA). Real-time PCR was performed using an ABI Power SYBR Green PCR Master Mix (ABI, Foster City, CA, USA) and 7900 HT Sequence Detection System (ABI, Foster City, CA, USA). Real-time PCR was performed with a 5-ng cDNA template using the $2 \times$ SYBR Green PCR buffer and $10-\mu \mathrm{mol}$ PCR primers in a total volume of $10 \mu \mathrm{l}$. Reactions were performed in 384-well PCR microplates. Additional file 1: Table S1 lists the primer sequences.

The expression of each lncRNA was represented as fold changes using the $\Delta \Delta$ Ct method to obtain quantitative results. Differences between groups were analyzed using a two-tailed Mann-Whitney $U$ test or unpaired $t$ test, based on the homogeneity of variance. Spearman's test was used for correlation studies. A value of $P<0.05$ was considered significant.

\section{Immunohistochemistry}

Immunohistochemical staining to detect $\mathrm{C}-\mathrm{X}-\mathrm{C}$ chemokine receptor type 4 (CXCR4), CD19, CD21, Toll-like receptor 9 (TLR9) and intercellular cell adhesion molecule 1 (ICAM1) was performed on LSG biopsy sections as described previously [25]. CXCR4 (ab124824, Abcam, Cambridge, MA, USA), CD3 (ab699, Abcam, Cambridge, MA, USA), CD19 (ab134114, Abcam, Cambridge, MA, USA), CD21 (ab75985, Abcam Cambridge, MA, USA), ICAM1 (ab53013, Abcam, Cambridge, MA, USA), CD20 (ab78237, Abcam, Cambridge, MA, USA), and TLR9 (BA3861-1, Boster Biotechnology, Wuhan, China) antibodies were used in this experiment. Negative control staining was performed by replacing primary antibodies with PBS. Positive immunoreactivity appeared as a brown color. Double staining for CD3 and CD20 was used to analyze $\mathrm{T} / \mathrm{B}$ cell segregation using the DouMaxvision ${ }^{\mathrm{Tm}}$ double-stain system (KIT-9998, Maixin Biotechnology, Fuzhou, China). A scoring system was used to describe the results of double staining as described previously [26]. The images of grade 1 to 3 are shown in Additional file 2: Figure S3.

\section{EBV-encoded RNA (EBER) in situ hybridization}

In situ hybridization was performed in LSG of $30 \mathrm{pSS}$ patients using Epstein-Barr Virus ISH Detection Kit 
(Triplex International Biosciences, Fuzhou, China), according to the manufacturer's protocol. Patients with lymphepithelioma were used as positive controls. The images of EBER-positive cells in representative ectopic lymphoid structures are shown in Additional file 3: Figure S4.

\section{Bioinformatics analysis}

Aberrantly expressed lncRNAs and mRNAs with statistical significance were identified using Volcano Plot filtering. The threshold used to screen up- or downregulated RNAs was a fold change $>2.0(P<0.05)$. Hierarchical clustering was performed in Cluster 3.0, and heat maps were generated in Java Treeview. The DE mRNAs or DE lncRNAs-related mRNAs were analyzed using pathway annotation and gene ontology (GO) functional enrichment in the Cytoscape 3.3 software. The gene-pathway network was also constructed using Cytoscape 3.3.

\section{Results}

Overview of aberrantly expressed IncRNAs and mRNAs in pSS

The expression levels of mRNAs and lncRNAs in four LSGs of pSS patients and paired control samples were analyzed using gene expression microarrays. The expression signatures of lncRNAs and mRNAs were reviewed using scatter plot and hierarchical clustering analyses. The scatter plots revealed that many lncRNAs and mRNAs were differentially expressed between pSS patients and control subjects (Fig. 1a, b). The heat maps of DE lncRNAs or mRNAs indicated the high level of concordance in pSS or control samples (Fig. 1c, d). The above-listed data indicated that changes in lncRNAs and mRNAs in LSGs were associated with the pathogenesis of pSS. Of the 63,431 lncRNAs and 39,887 mRNAs in the microarray, 1243 lncRNAs and 1457 mRNAs were significantly differentially expressed in the labial glands
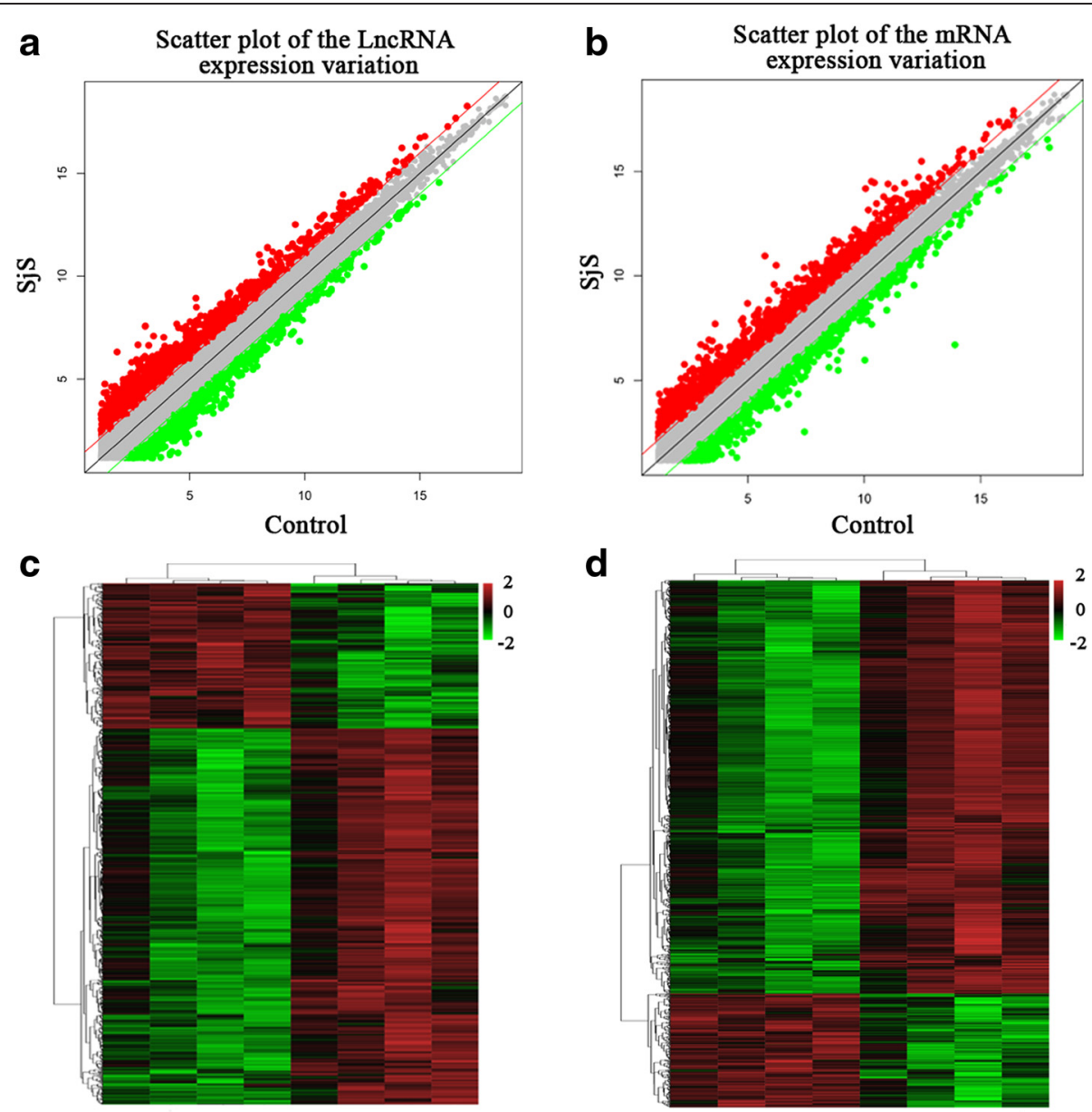

d

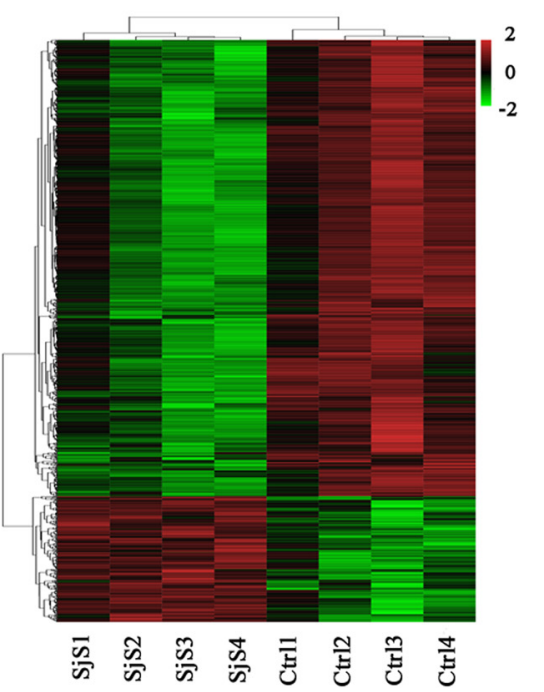

Fig. 1 The expression profiling of IncRNA and mRNA in LSGs of pSS patients and healthy controls. a, b Scatter plot comparing global IncRNA or mRNA gene expression profiles between pSS and control subjects. Green lines and red line indicate twofold differences in either direction in IncRNA and mRNA expression. c, d Heat map showing hierarchical clustering of IncRNAs or mRNA with expression changes greater than twofold. Red and green colors represent up- and downregulated genes, respectively. IncRNA long noncoding RNA, SjS Sjögren's syndrome 
of pSS patients compared to control subjects (fold change $>2, P<0.05)$. A total of 890 lncRNAs and 1141 mRNAs were upregulated, and 353 lncRNAs and 316 mRNAs were downregulated. Table 2 lists the 20 most up- and downregulated DE lncRNAs. Table 3 lists the 20 most up- and downregulated DE mRNAs. All of the data from the microarray trials are stored in the GEO database with accession number GSE76013.

\section{Real-time PCR: IncRNA expression profiles and correlations with clinical characteristics}

Aberrantly expressed lncRNAs were identified in labial gland tissue samples from 30 pSS patients and 16 control subjects. The selection of representative lncRNAs for validation was based on the two approaches described in the Methods section. Nine lncRNAs were selected: NR_002712, n341833, lnc-UTS2D-1:1, TCONS_12_00014794, n336161, ENST00000420219.1, ENST00000455309.1, n340599, and ENST00000546086.1. The results demonstrated that ENST00000420219.1 (3.13-fold), ENST00000455309.1 (2.51-fold), n336161 (2.45-fold), NR_002712 (2.41-fold), ENST00000546086.1 (1.94-fold), Lnc-UTS2D-1:1 (1.79fold), n340599 (1.69-fold), and TCONS_12_00014794 (1.28-fold) were significantly increased in pSS (Fig. 2). However, n341833 was not dysregulated in pSS patients.
The next step was an analysis to determine whether any correlations existed between these DE IncRNA expression levels and the clinical characteristics. Figures $3 a-d$ and $4 a-b$ show that strong correlations were observed in eight pairs: (1) disease course with six lncRNA expression levels; (2) visual analogue scale (VAS) of parotid swelling with two lncRNAs; (3) VAS of dry eyes with one lncRNA; (4) $\beta 2$ microglobulin with six lncRNAs; (5) erythrocyte sedimentation rate (ESR) with three lncRNAs; (6) rheumatoid factor (RF) with two lncRNAs; (7) immunoglobulin (Ig)A with two lncRNAs; and (8) IgM with one lncRNA. Four lncRNAs were significantly upregulated in SSB-positive patients compared to SSB-negative patients (Fig. 4c-d). No significant correlation existed between these DE lncRNAs and dry mouth, IgE, IgG, complement 3 (C3), complement 4 (C4), C-reactive protein (CRP) and grading of labial biopsy. Additional file 4: Table $\mathrm{S} 2$ shows the detailed correlation analysis results. A multivariate model was used to identify lncRNAs that correlated independently with disease characteristics. Additional file 5: Table S3 shows the results of this analysis.

\section{Functional prediction of DE mRNAs}

GO and pathway analyses of the up- and downregulated genes in LSGs of pSS patients were performed to further

Table 2 Top 20 significantly differential expressed IncRNAs between pSS patients and control subjects

\begin{tabular}{|c|c|c|c|c|c|}
\hline \multicolumn{3}{|l|}{ Upregulated } & \multicolumn{3}{|l|}{ Downregulated } \\
\hline IncRNAs & $P$ value & Fold change & IncRNAs & $P$ value & Fold change \\
\hline Inc-UTS2D-1:1 & 0.001 & 24.377 & NR_026839.1 & 0.010 & -7.135 \\
\hline NR_002712 & 0.018 & 20.057 & TCONS_12_00013113 & 0.007 & -6.244 \\
\hline n341833 & 0.001 & 16.933 & NR_026842.1 & 0.022 & -5.943 \\
\hline Inc-MMP3-1:1 & 0.023 & 11.056 & TCONS_12_00004830 & 0.033 & -5.319 \\
\hline Inc-LGALS14-1:2 & 0.000 & 9.526 & NR_046443.1 & 0.033 & -5.280 \\
\hline NR_034176.1 & 0.005 & 8.737 & Inc-CYR61-2:1 & 0.008 & -4.783 \\
\hline n333136 & 0.026 & 7.420 & NR_037839 & 0.001 & -4.760 \\
\hline n336199 & 0.031 & 6.893 & NR_037839 & 0.022 & -4.747 \\
\hline NR_073198.1 & 0.012 & 6.880 & n333443 & 0.031 & -4.633 \\
\hline Inc-MAGEA12-2:1 & 0.013 & 6.866 & NR_026838 & 0.012 & -4.358 \\
\hline Inc-ADAM2-1:1 & 0.020 & 6.853 & NR_026838.1 & 0.025 & -4.358 \\
\hline n337610 & 0.005 & 6.654 & TCONS_12_00019075 & 0.006 & -4.350 \\
\hline n334829 & 0.007 & 6.628 & ENST00000495382.1 & 0.014 & -4.313 \\
\hline ENST00000577557.1 & 0.024 & 6.626 & NR_036580 & 0.028 & -4.308 \\
\hline XR_111691 & 0.010 & 6.482 & Inc-OBP2B-2:1 & 0.011 & -4.294 \\
\hline XR_111691 & 0.011 & 6.384 & n333418 & 0.003 & -4.256 \\
\hline Inc-KATNAL1-3:14 & 0.009 & 6.336 & ENST00000508179.1 & 0.005 & -4.180 \\
\hline n336575 & 0.017 & 6.215 & n334464 & 0.004 & -4.117 \\
\hline ENST00000578280.1 & 0.013 & 6.183 & TCONS_12_00010194 & 0.022 & -4.005 \\
\hline Inc-BRD1-4:1 & 0.002 & 6.101 & Inc-ZNF572-1:15 & 0.012 & -3.962 \\
\hline
\end{tabular}

IncRNA long noncoding RNA, SjS Sjögren's syndrome 
Table $\mathbf{3}$ Top 20 significantly differential expressed mRNAs between pSS patients and control subjects

\begin{tabular}{|c|c|c|c|c|c|}
\hline \multicolumn{3}{|c|}{ Upregulated } & \multicolumn{3}{|l|}{ Downregulated } \\
\hline mRNAs & $P$ value & Fold change & mRNAs & $P$ value & Fold change \\
\hline$\overline{\text { EGFL6 }}$ & 0.018 & 26.237 & ITLN1 & 0.003 & -104.642 \\
\hline SCGB1D2 & 0.020 & 17.197 & $\mathrm{TRH}$ & 0.001 & -26.423 \\
\hline SCGB2A2 & 0.033 & 16.941 & C4orf40 & 0.036 & -9.826 \\
\hline CXCL13 & 0.018 & 16.911 & LCE5A & 0.001 & -7.139 \\
\hline SCGB1D1 & 0.019 & 15.752 & CYP2F1 & 0.013 & -6.398 \\
\hline FCRL4 & 0.010 & 14.227 & FXYD2 & 0.000 & -6.070 \\
\hline MMP12 & 0.028 & 11.131 & CADPS & 0.002 & -5.823 \\
\hline IDO1 & 0.024 & 9.307 & ASTN1 & 0.038 & -5.735 \\
\hline CD1A & 0.007 & 8.942 & CABS1 & 0.022 & -5.372 \\
\hline CXCL11 & 0.018 & 8.175 & GABRG3 & 0.005 & -5.303 \\
\hline KLHDC7B & 0.008 & 8.152 & FGF12 & 0.039 & -5.231 \\
\hline CXCL9 & 0.028 & 7.876 & CSN3 & 0.008 & -4.829 \\
\hline TIMD4 & 0.001 & 7.647 & $\mathrm{DACH} 2$ & 0.048 & -4.709 \\
\hline UTS2D & 0.029 & 7.368 & LEFTY1 & 0.027 & -4.709 \\
\hline SLC26A4 & 0.014 & 7.037 & NA & 0.048 & -4.652 \\
\hline ZBTB32 & 0.011 & 6.793 & XLOC_002852 & 0.011 & -4.508 \\
\hline TIMD4 & 0.004 & 6.746 & NA & 0.012 & -4.375 \\
\hline EXOC3L4 & 0.003 & 6.281 & PPP1R17 & 0.033 & -4.332 \\
\hline IFNG & 0.018 & 6.201 & $A B O$ & 0.012 & -4.272 \\
\hline CTLA4 & 0.011 & 6.177 & SLC35G1 & 0.024 & -4.215 \\
\hline
\end{tabular}

SjS Sjögren's syndrome

examine the potential mechanism of pSS. The GO results indicated that the most significantly enriched cellular components of upregulated mRNAs in LSGs of pSS were the external side of the plasma membrane, immunological synapse, and MHC class II protein complex (Fig. 5a). The most significantly enriched molecular functions of upregulated mRNAs were peptide antigen binding, MHC class II receptor activity, and transmembrane signaling receptor activity (Fig. 5a). The most significantly enriched biological processes of upregulated mRNAs were immune response, inflammatory response, and cytokine-mediated signaling pathway (Fig. 5a). The most significantly enriched cellular components of downregulated mRNAs in LSGs of pSS were host cell nucleus, nucleus, and nucleolus (Fig. 5b). The most significantly enriched molecular functions of downregulated mRNAs were heparanase activity, monooxygenase activity, and mu-type opioid receptor binding (Fig. 5b). The most significantly enriched biological processes of downregulated mRNAs were transmembrane transport, mammary gland alveolus development, and potassium ion homeostasis (Fig. 5b). DE mRNAs were analyzed in the Kyoto Encyclopedia of Genes and Genomes (KEGG).
The results revealed that the upregulated mRNAs in LSGs of pSS were significantly involved in graft-versushost disease, cytokine-cytokine receptor interactions, and cell adhesion molecules (Fig. 5c). The downregulated mRNAs were significantly involved in gastric acid secretion, mineral absorption, and retinol metabolism (Fig. 5d).

\section{Relational analyses of IncRNAs and mRNAs}

Twenty-eight DE mRNAs from the co-expression network data strongly correlated with the eight validated lncRNAs $(P<0.001, r>0.99)$. Table 4 shows the detailed information of these mRNAs. Figure 6 shows that genepathway network graph analyses revealed that the $28 \mathrm{DE}$ mRNAs, including ICAM1, TLR9, TNF receptorassociated factor 1 (TRAF1), CXCR4, chemokine (C-C motif) ligand 20 (CCL20), and CD19 were likely involved in chemokine signaling pathways, the nuclear factor-kappa B (NF-Kb) signaling pathway, TNF signaling pathway, African trypanosomiasis, natural killer cellmediated cytotoxicity, and Epstein-Barr virus infection [27-30], which are involved in the pathogenesis of pSS. Additional file 6: Figures S1 and Additional file 7: Figure S2 show that the results of immunohistochemistry in LSGs of pSS patients confirmed the overexpression of CD19, CXCR4, ICAM1, and TLR9 proteins. These results indicate the functional roles of DE IncRNAs in the progress of pSS. Since many of these lncRNA are supposed to regulate immune functions, the correlation between the eight upregulated IncRNAs and main histopathological data, such as the infection of Epstein-Barr virus, B/T cell segregation, and presence/ absence of ectopic lymphoid structures, were analyzed. The results revealed no significant correlation exists (Additional file 8: Figure S5).

\section{Discussion}

LncRNAs were primarily investigated in genomic imprinting, cancers, and cell differentiation, but these molecules are emerging as important regulators of immune cell differentiation and the activation of innate immunity. However, the identification of lncRNA expression in autoimmune diseases is largely underexplored. Few studies reported that IncRNAs played a crucial role in autoimmune diseases, such as SLE, RA, T1DM and MS [31-36], but fewer studies have examined the expression profile of lncRNAs in pSS. The critical role of IncRNAs in the pathogenesis of pSS was demonstrated previously [37]. Therefore, differentially expressed lncRNAs in the LSGs of pSS patients were identified using microarray experiments.

The results demonstrated that upregulated mRNAs were far more numerous than downregulated mRNAs in pSS samples, which indicated the activation of many 


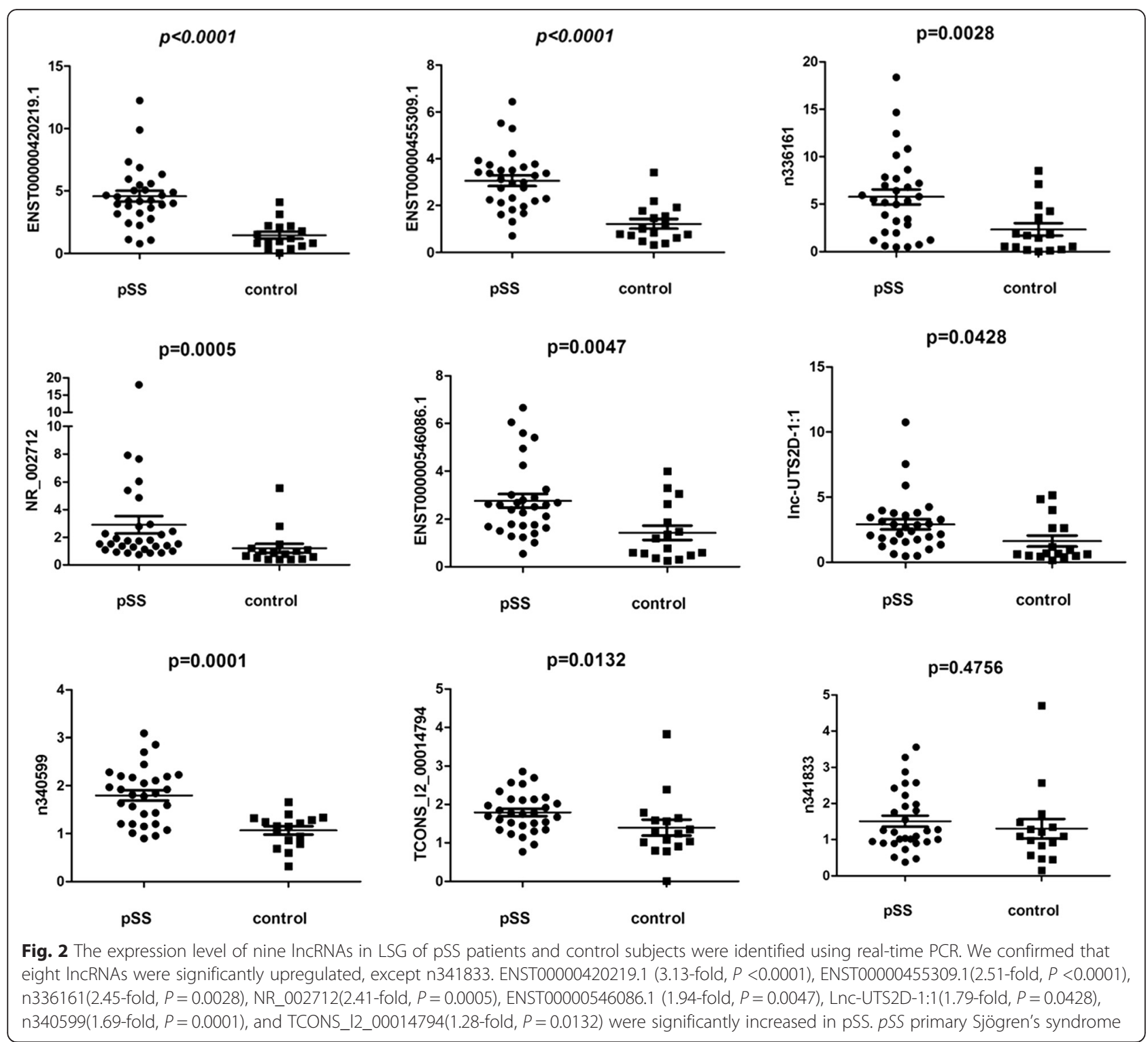

new biological processes or signaling pathways in pathological conditions. DE mRNAs were further analyzed in pSS patients using GO term enrichment and pathway enrichment analyses. The GO results indicated that the most significantly enriched cellular components of upregulated mRNAs in LSGs of pSS patients were the external side of plasma membrane, immunological synapse, and MHC class II protein complex. The most significantly enriched molecular functions of upregulated mRNAs were peptide antigen binding, MHC class II receptor activity, and transmembrane signaling receptor activity. These results are consistent with previous studies that demonstrated aberrantly expressed MHC class II and costimulation molecules in the epithelial cells of salivary glands in pSS patients $[38,39]$. The abnormal expression of these molecules on the surface of salivary gland epithelial cells may favor the presentation of SSA and SSB epitopes to $\mathrm{T}$ cells and lead to autoantibody production [40]. The immune response, inflammatory response, and cytokine-mediated signaling pathway were the most significantly enriched biological processes of the upregulated mRNAs. The results suggest that an active autoimmune inflammatory response occurred in the epithelial cells of salivary glands in pSS patients. The results also demonstrated that several cellular components, molecular functions, and biological processes were inhibited. The pathway analysis results suggested that the upregulated mRNAs in LSGs of pSS patients were significantly involved in graft-versus-host diseases, cytokine-cytokine receptor interactions, and cell 


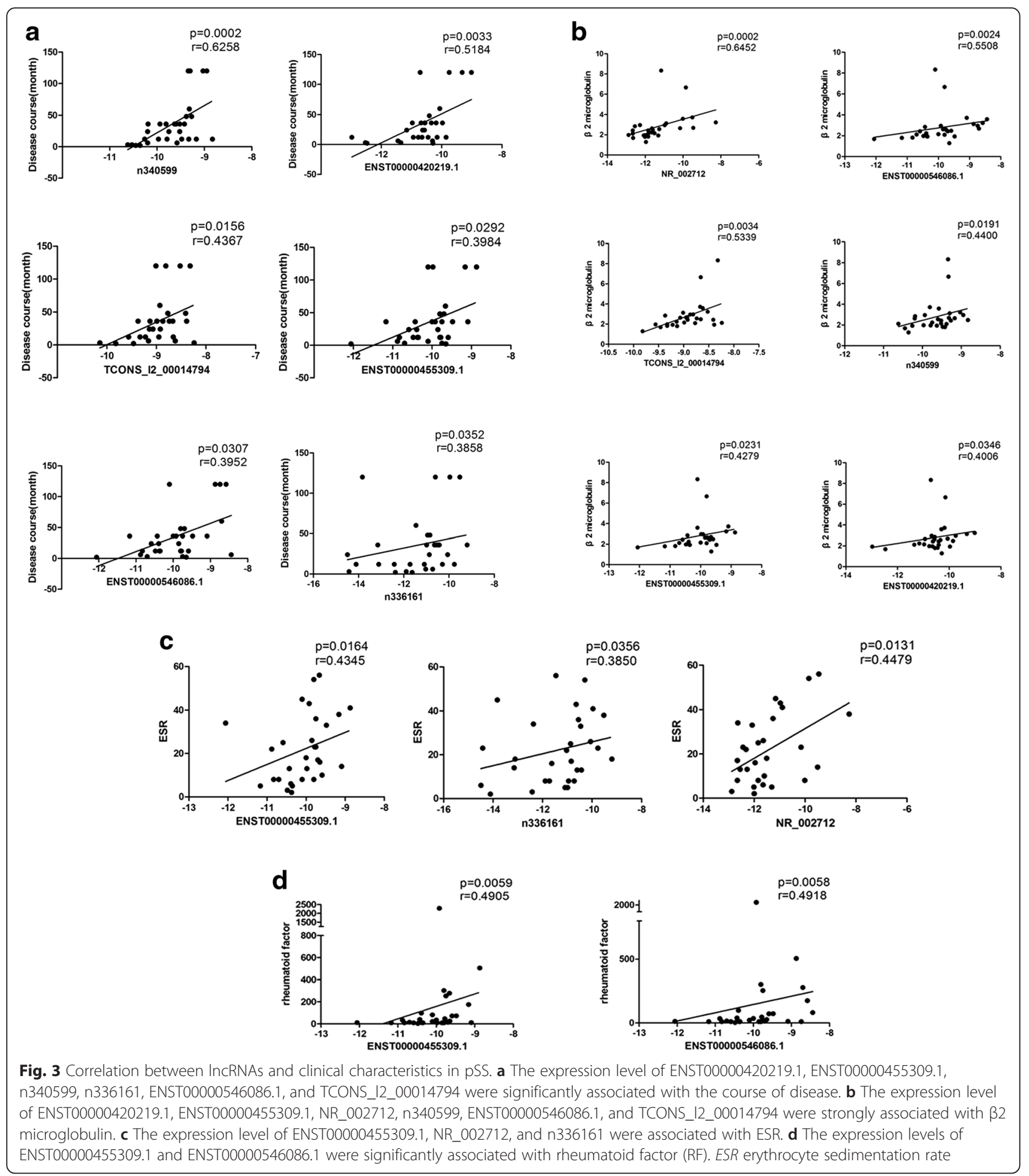

adhesion molecules. The results also suggest that deficiencies occurred in the labial gland epithelial cells of pSS patients and ended with the activation of autoimmune inflammation and the release of inflammatory cytokines by immune cells.
Nine of the aberrantly expressed lncRNAs, including NR_002712, n341833, Inc-UTS2D-1:1, TCONS_12_00014794, n336161, ENST00000420219.1, ENST00000455309.1, n340599, and ENST00000546086.1, were further confirmed in the $30 \mathrm{pSS}$ patients using real-time PCR. 


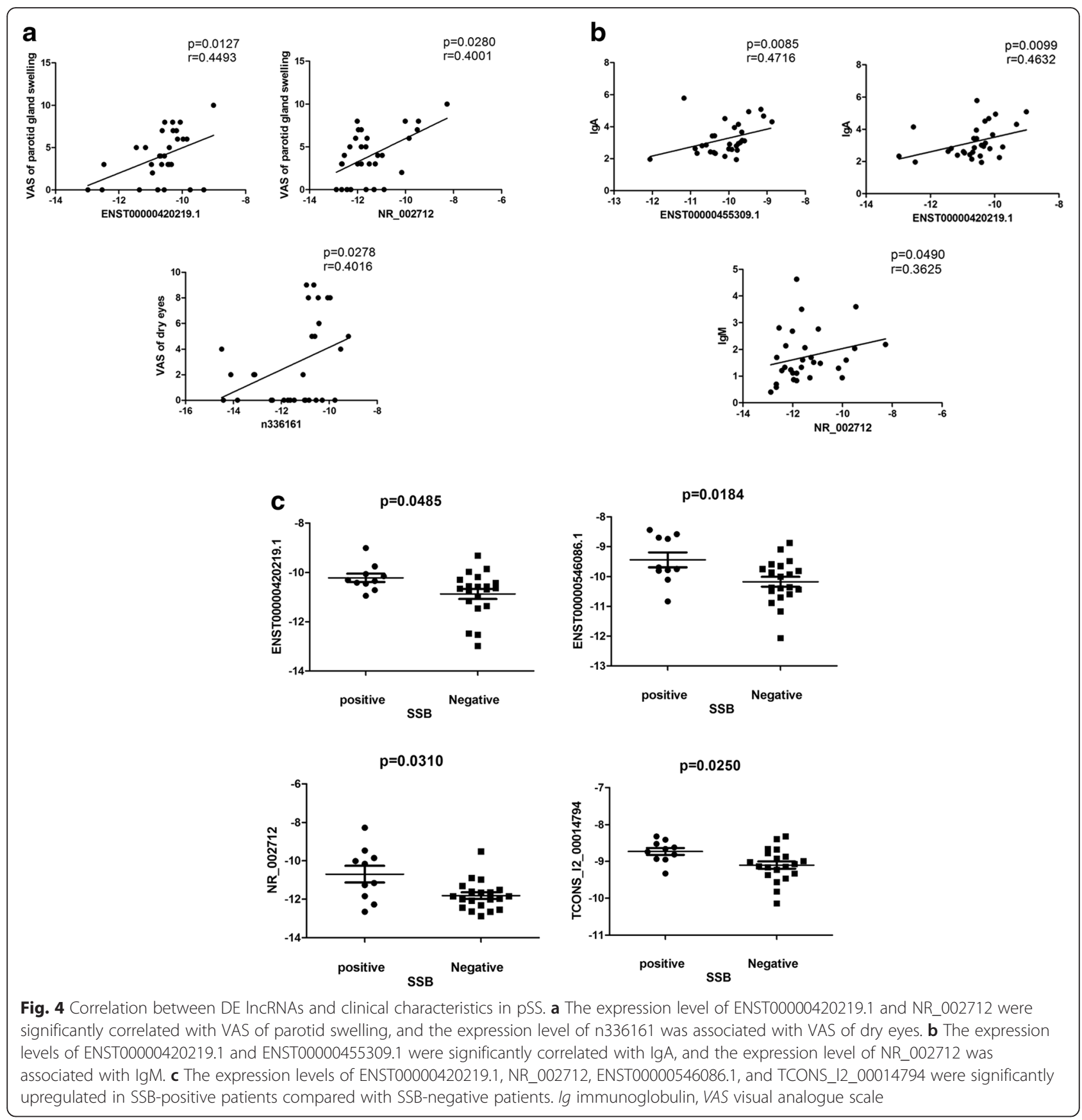

Eight lncRNAs were significantly upregulated, but n341833 was not upregulated. Recent prospective studies confirmed that serum $\beta 2$ microglobulin levels were associated with the EULAR Sjögren's syndrome disease activity index (ESSDAI) and EULAR SS patient-reported index (ESSPRI) in the pSS patients $[41,42]$. Notably, the expression levels of NR 002712, ENST00000546086.1, TCONS_12_00014794, n340599, ENST00000455309.1, and ENST00000420219.1 correlated with the $\beta 2$ microglobulin levels, which suggest that the upregulated expression of these lncRNAs was associated with active disease states. These lncRNAs may be strongly involved in the progress of pSS, especially ENST00000455309.1, which significantly correlated with the disease course, ESR, RF, and IgA expression levels. These multiple correlations were also observed with other lncRNAs. The results further confirmed that these lncRNAs played critical roles in the pathophysiology of pSS, but further functional studies are needed to examine the potential mechanisms. The regulatory mechanisms and functional principles of lncRNAs were elucidated recently. 


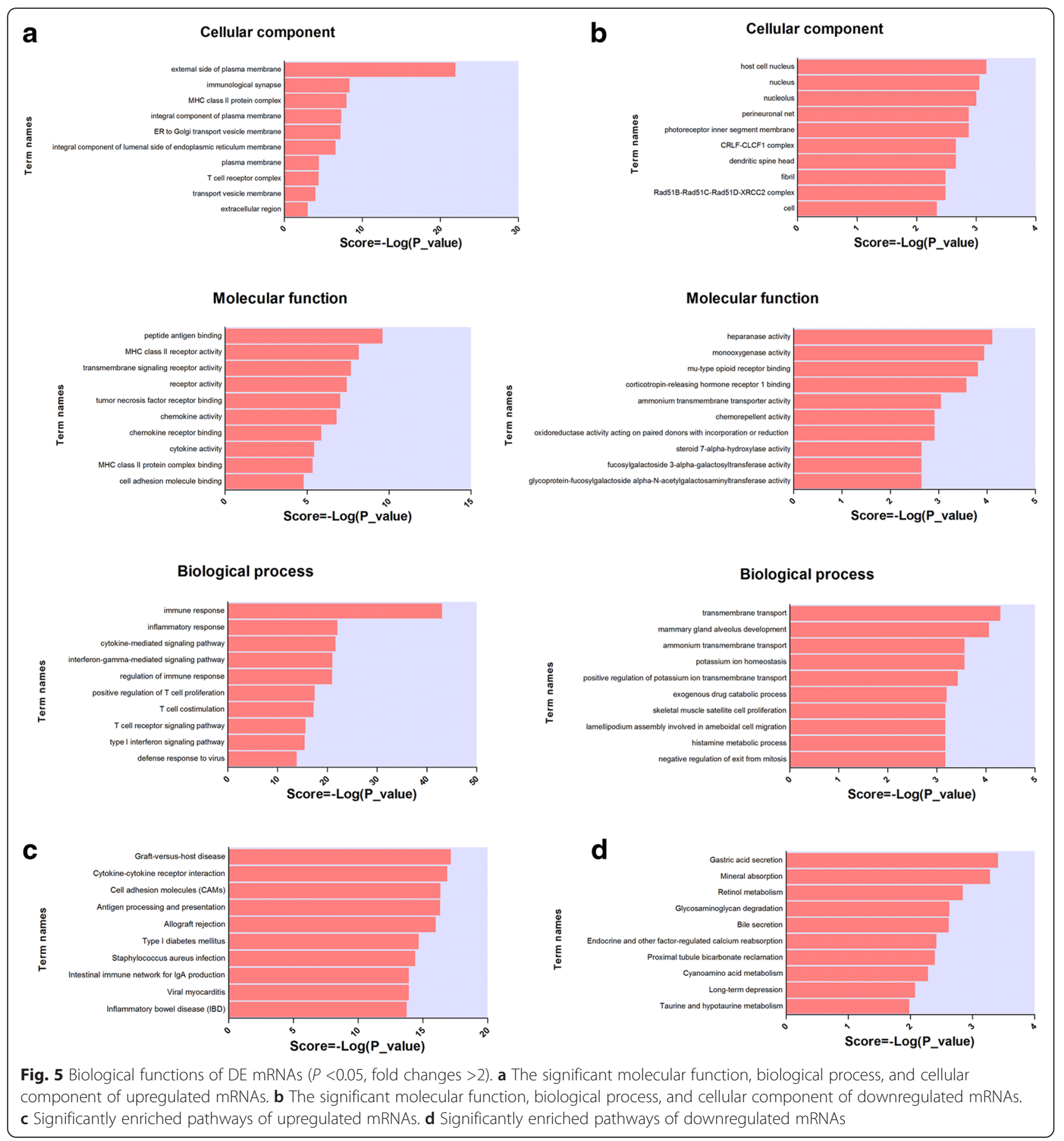

LncRNAs that regulate the abundance of genomically neighboring or distal gene products are classified as cis-regulatory models or trans-regulatory models, respectively [43]. However, IncRNAs achieve regulatory functions via modularity, the collection of diverse combinations of proteins, and possible RNA or DNA interactions. This study investigated the potential targets of eight DE lncRNAs by using an lncRNA-
mRNA co-expression network. This network revealed that 28 DE mRNAs were strongly associated with eight DE lncRNAs. A gene-pathway network of the eight DE lncRNA-related genes was established to explore the functional mechanism. The results revealed a significant involvement of these eight DE lncRNAs in several important signaling pathways that play crucial roles in the pathogenesis of pSS. However, the 
Table 4 Detailed information of DE IncRNAs linked DE mRNA

\begin{tabular}{|c|c|c|c|c|c|c|}
\hline mRNA gene symbol & $P$ value & Fold change & Regulation & Correlated IncRNA gene name & $r$ value & $P$ value \\
\hline $\mathrm{ACY} 3$ & 0.028 & 2.221 & up & n340599 & 0.9996 & 0.0004 \\
\hline AGAP2 & 0.016 & 2.618 & up & $\begin{array}{l}\text { ENST00000420219.1, } \\
\text { n336161 }\end{array}$ & $\begin{array}{l}0.9995 \\
0.9992\end{array}$ & $\begin{array}{l}0.0005 \\
0.0008\end{array}$ \\
\hline ARHGAP30 & 0.019 & 2.377 & up & $\begin{array}{l}\text { n336161, } \\
\text { ENST00000455309.1 }\end{array}$ & $\begin{array}{l}0.9993 \\
0.9991\end{array}$ & $\begin{array}{l}0.0007 \\
0.0009\end{array}$ \\
\hline CCL20 & 0.013 & 4.295 & up & TCONS_12_00014794 & 0.9991 & 0.0009 \\
\hline CCR5 & 0.003 & 4.040 & up & ENST00000455309.1 & 0.9993 & 0.0007 \\
\hline CD19 & 0.033 & 5.657 & up & n340599 & 0.9992 & 0.0008 \\
\hline CD6 & 0.002 & 2.604 & up & ENST00000420219.1 & 09990 & 0.0009 \\
\hline CFP & 0.011 & 2.089 & up & $\begin{array}{l}\text { ENST00000546086.1, } \\
\text { TCONS_I2_00014794 }\end{array}$ & $\begin{array}{l}0.9995 \\
0.9996\end{array}$ & $\begin{array}{l}0.0005 \\
0.0004\end{array}$ \\
\hline CXCR4 & 0.028 & 5.527 & up & $\begin{array}{l}\text { ENST000000546086.1, } \\
\text { TCONS_I2_00014794 }\end{array}$ & $\begin{array}{l}0.9998 \\
0.9997\end{array}$ & $\begin{array}{l}0.0002 \\
0.0003\end{array}$ \\
\hline CYTH4 & 0.015 & 2.151 & up & n340599 & 0.9996 & 0.0004 \\
\hline GIMAP4 & 0.004 & 2.905 & up & ENST00000455309.1 & 0.9997 & 0.0003 \\
\hline GNGT2 & 0.011 & 3.857 & up & $\begin{array}{l}\text { ENST00000455309.1, } \\
\text { n336161 }\end{array}$ & $\begin{array}{l}0.9991 \\
0.9999\end{array}$ & $\begin{array}{l}0.0009 \\
0.0001\end{array}$ \\
\hline HCST & 0.005 & 3.011 & up & $\begin{array}{l}\text { ENST000000546086.1, } \\
\text { TCONS_I2_00014794 }\end{array}$ & $\begin{array}{l}0.9998 \\
0.9995\end{array}$ & $\begin{array}{l}0.0002 \\
0.0005\end{array}$ \\
\hline HIST1H2Al & 0.033 & 3.800 & up & ENST00000455309.1 & 0.9995 & 0.0005 \\
\hline ICAM1 & 0.008 & 2.385 & up & $\begin{array}{l}\text { ENST00000455309.1 } \\
\text { n336161 }\end{array}$ & $\begin{array}{l}0.9991 \\
0.9994\end{array}$ & $\begin{array}{l}0.0009 \\
0.0006\end{array}$ \\
\hline KIF20B & 0.007 & 2.118 & up & ENST00000455309.1, & 0.9992 & 0.0008 \\
\hline LAPTM5 & 0.022 & 3.223 & up & ENST00000546086.1 & 0.9997 & 0.0003 \\
\hline LEF1 & 0.020 & 3.743 & up & TCONS_12_00014794 & 0.9999 & 0.0001 \\
\hline LILRB1 & 0.003 & 3.180 & up & $\begin{array}{l}\text { ENST00000420219.1, } \\
\text { ENST00000455309.1, } \\
\text { n336161 }\end{array}$ & $\begin{array}{l}0.9994 \\
0.9998 \\
0.9997\end{array}$ & $\begin{array}{l}0.0006 \\
0.00020 .0003\end{array}$ \\
\hline PRKCQ & 0.025 & 2.969 & up & ENST00000455309.1 & 0.9994 & 0.0006 \\
\hline RABGAP1L & 0.010 & 2.149 & up & n340599 & 0.9997 & 0.0003 \\
\hline RAC2 & 0.006 & 3.169 & up & n340599 & 0.9992 & 0.0008 \\
\hline RLTPR & 0.016 & 2.094 & up & $\begin{array}{l}\text { ENST00000420219.1, } \\
\text { n336161 }\end{array}$ & $\begin{array}{l}0.9994 \\
0.9993\end{array}$ & $\begin{array}{l}0.0006 \\
0.0007\end{array}$ \\
\hline SUSD3 & 0.010 & 2.587 & up & $\begin{array}{l}\text { ENST00000546086.1, } \\
\text { TCONS_I2_00014794 }\end{array}$ & $\begin{array}{l}0.9998 \\
0.9999\end{array}$ & $\begin{array}{l}0.0002 \\
0.0001\end{array}$ \\
\hline TLR9 & 0.043 & 2.029 & up & n340599 & 0.9992 & 0.0008 \\
\hline TRAF1 & 0.014 & 2.530 & up & n340599 & 0.9998 & 0.0002 \\
\hline WDFY4 & 0.014 & 3.251 & up & n340599 & 0.9995 & 0.0005 \\
\hline ZNF831 & 0.014 & 4.663 & up & ENST00000455309.1 & 0.9997 & 0.0003 \\
\hline
\end{tabular}

$D E$ differentially expressed, IncRNA long noncoding RNA

precise regulatory mechanism of lncRNAs requires further study. Animal models should be used in further research to help elucidate the biological processes of lncRNAs in pSS. Another limitation of this study was the lack of pSS mouse models. LncRNAs expression profiles should be detected in different animal models because of the variety of pSS mouse models.

\section{Conclusions}

This study revealed aberrant expression profiles of lncRNAs in LSGs of pSS patients for the first time. A total of 1243 lncRNAs and 1457 mRNAs were differentially expressed in the labial glands of pSS patients compared to the control subjects, and eight lncRNAs were further confirmed using real-time PCR in 30 pSS patients. Strong correlations between lncRNAs and clinical 


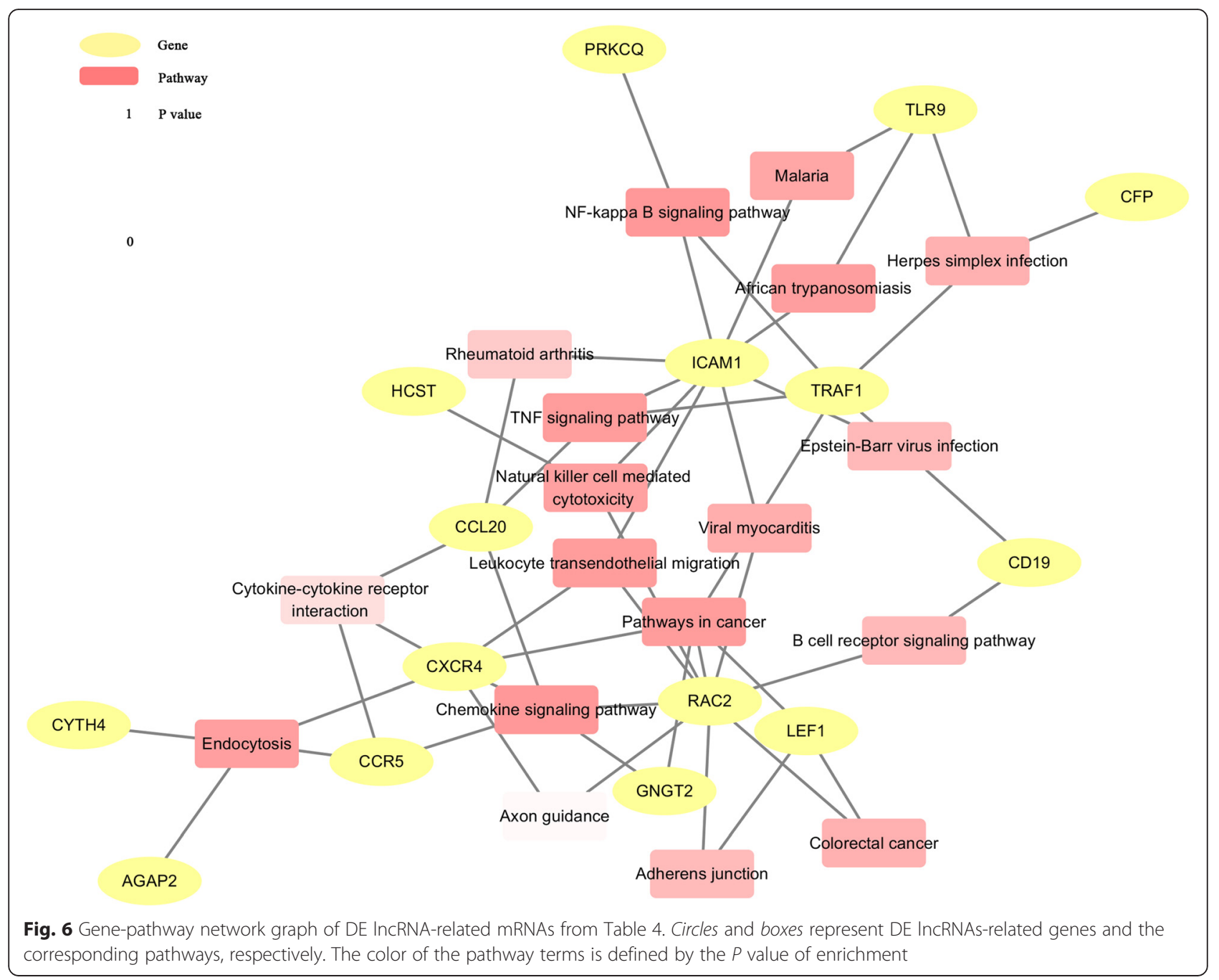

characteristics were observed. This study will lead to novel directions in pSS diagnosis and therapy.

\section{Ethics approval and consent to participate}

This study was approved by the Ethics Committee, Faculty of Medicine, Shanghai Jiao Tong University.

\section{Additional files}

Additional file 1: Table S1. Primer sequences used in validation of IncRNAs. (DOCX $14 \mathrm{~kb}$ )

Additional file 2: Figure S3. Characterization of the cellular infiltrate and histomorphologic grading of LSG. (A) Shown are representative examples of grade 1 ( $\mathrm{G} 1 ;<50$ periductal lymphocytes); $(\mathrm{B}, \mathrm{C})$ grade 2 ( $G 2 ;>50$ periductal lymphocytes): nonsegregated and segregated aggregates (NS-G2 and S-G2, respectively); (D,F) grade $3(\mathrm{G} 3 ;>50$ periductal lymphocytes, with GC-like structures). (TIF 58923 kb)

Additional file 3: Figure S4. EBER expression in LSG of pSS patients. (A) Significant number of EBER+ cells in representative ectopic lymphoid structure-positive LSG tissue. (B) Double staining for CD3 and CD20 in the same section of LSG tissue. (C) Staining for CD21 in the same section of LSG tissue. (TIF $53011 \mathrm{~kb}$ )

Additional file 4: Table S2. Detailed correlation analysis results. (DOCX 20 kb)

Additional file 5: Table S3. Multivariate model used to identify the IncRNAs that correlated independently with disease characteristics. (DOCX $14 \mathrm{~kb}$ )

Additional file 6: Figure S1. Immunohistochemistry for CD19 and ICAM1 in LSG of pSS and healthy control. (A, E) Infiltrating lymphocytes in LSG of pSS were positively staining for CD19 (200x and 400x magnification); (B, F) glandular epithelial cells from control were negative (200x and 400× magnification); (C, G) infiltrating lymphocytes and adjacent glandular epithelial cells in LSG of pSS were positively staining for ICAM1 (200x and 400× magnification). (D, H) Glandular epithelial cells from control were negative (200× and 400× magnification). (TIF $28780 \mathrm{~kb}$ )

Additional file 7: Figure S2. Immunohistochemistry for TLR9 and CXCR4 in LSG of pSS and healthy controls. (A, E) Infiltrating lymphocytes in LSG of pSS positively stained for TLR9 (200x and 400x magnification); (B, F) glandular epithelial cells from control were negative (200x and $400 \times$ magnification); (C, G) infiltrating lymphocytes and adjacent glandular epithelial cells in LSG of pSS were positively stained for CXCR4 (200x and 400x magnification). (D, H) Glandular epithelial cells from control were negative (200x and 400x magnification). (TIF $28419 \mathrm{~kb}$ ) 
Additional file 8: Figure S5. Expression level analysis between the eight upregulated IncRNAs and main histopathological data. (A) Expression of eight IncRNAs in grade 1 (G1), nonsegregated grade 2 (NS-G2), segregated grade 2 (S-G2), and grade 3 (G3) foci in LSG from 30 patients with pSS. (B) Expression of eight IncRNAs in EBER-positive and -negative LSG from 30 patients with pSS. (TIF $2332 \mathrm{~kb}$ )

\section{Abbreviations}

C3: complement 3; C4: complement 4; CCL20: chemokine (C-C motif) ligand 20; CRP: C-reactive protein; CXCR4: C-X-C chemokine receptor type 4; DE: differentially expressed; EBER: EBV-encoded RNA; ESR: erythrocyte sedimentation rate; ESSDAl: EULAR Sjögren's syndrome disease activity index; ESSPRI: EULAR SS patient-reported index; GO: gene ontology; ICAM1: intercellular cell adhesion molecule 1; Ig: immunoglobulin; IncRNA: long noncoding RNA; LSG: labial salivary gland; MS: multiple sclerosis; pSS: primary Sjögren's syndrome; RA: rheumatoid arthritis; RF: rheumatoid factor; SLE: systemic lupus erythematosus; T1DM: type I diabetes mellitus; TLR9: Toll-like receptor 9; TNF-a: tumor necrosis factor alpha; TRAF1: TNF receptor-associated factor 1; VAS: visual analogue scale.

\section{Competing interests}

The authors declare that they have no competing interests.

\section{Authors' contributions}

HS carried out the molecular genetic studies, participated in the sequence alignment, and drafted the manuscript. NC carried out the molecular genetic studies, the immunoperoxidase assay, and revised the manuscript. LX participated in the sequence alignment, collected tissue samples, and helped to draft the manuscript. YP carried out in situ hybridization experiment and collected tissue samples. LZ participated in the design of the study, performed the statistical analysis, and helped to revise the manuscript. CY conceived of the study, and participated in its design and coordination, and helped to draft the manuscript. All authors read and approved the final manuscript.

\section{Acknowledgements}

This work is supported by Key Project of Shanghai Municipal Commission of Health and Family Planning (Project Number: 2014035), Science and Technology Commission of Shanghai Municipality (Project Number: 16ZR1419000).

\section{Received: 5 February 2016 Accepted: 26 April 2016}

\section{Published online: 17 May 2016}

\section{References}

1. Fox RI. Sjogren's syndrome. Lancet. 2005;366(9482):321-31.

2. Gajewski TF, Schreiber H, Fu YX. Innate and adaptive immune cells in the tumor microenvironment. Nat Immunol. 2013;14(10):1014-22.

3. Gierut A, Perlman H, Pope RM. Innate immunity and rheumatoid arthritis. Rheum Dis Clin North Am. 2010;36(2):271-96.

4. Moore KJ, Sheedy FJ, Fisher EA. Macrophages in atherosclerosis: a dynamic balance. Nat Rev Immunol. 2013;13(10):709-21.

5. Li Y, Shi X. MicroRNAs in the regulation of TLR and RIG-I pathways. Cell Mol Immunol. 2013;10(1):65-71.

6. O'Connell RM, Rao DS, Baltimore D. microRNA regulation of inflammatory responses. Annu Rev Immunol. 2012;30:295-312.

7. Fitzgerald KA, Caffrey DR. Long noncoding RNAs in innate and adaptive immunity. Curr Opin Immunol. 2014;26:140-6.

8. Heward JA, Lindsay MA. Long non-coding RNAs in the regulation of the immune response. Trends Immunol. 2014;35(9):408-19.

9. Sauvageau M, Goff LA, Lodato S, Bonev B, Groff AF, Gerhardinger C, et al. Multiple knockout mouse models reveal lincRNAs are required for life and brain development. eLife. 2013;2:e01749.

10. Carpenter S, Aiello D, Atianand MK, Ricci EP, Gandhi P, Hall LL, et al. A long noncoding RNA mediates both activation and repression of immune response genes. Science. 2013;341(6147):789-92.

11. Szmyrka-Kaczmarek M, Kosmaczewska A, Ciszak L, Szteblich A, Wiland P. Peripheral blood Th17/Treg imbalance in patients with low-active systemic lupus erythematosus. Postepy Hig Med Dosw (Online). 2014;68:893-8.

12. Imamura K, Imamachi N, Akizuki G, Kumakura M, Kawaguchi A, Nagata K et al. Long noncoding RNA NEAT1-dependent SFPQ relocation from promoter region to paraspeckle mediates IL8 expression upon immune stimuli. Mol Cell. 2014:53(3):393-406.

13. Hirose T, Virnicchi G, Tanigawa A, Naganuma T, Li R, Kimura H, et al. NEAT1 long noncoding RNA regulates transcription via protein sequestration within subnuclear bodies. Mol Biol Cell. 2014;25(1):169-83.

14. Cui H, Xie N, Tan Z, Banerjee S, Thannickal VJ, Abraham E, et al. The human long noncoding RNA Inc-IL7R regulates the inflammatory response. Eur J Immunol. 2014;44(7):2085-95.

15. Krawczyk M, Emerson BM. p50-associated COX-2 extragenic RNA (PACER) activates COX-2 gene expression by occluding repressive NF-kappaB complexes. eLife. 2014;3:e01776.

16. Wang $P$, Xue $Y$, Han $Y$, Lin L, Wu C, Xu S, et al. The STAT3-binding long noncoding RNA Inc-DC controls human dendritic cell differentiation. Science. 2014;344(6181):310-3.

17. Ilott NE, Heward JA, Roux B, Tsitsiou E, Fenwick PS, Lenzi L, et al. Long noncoding RNAs and enhancer RNAs regulate the lipopolysaccharide-induced inflammatory response in human monocytes. Nat Commun. 2014:5:3979.

18. Chan J, Atianand M, Jiang Z, Carpenter S, Aiello D, Elling R, et al. Cutting edge: a natural antisense transcript, AS-IL1alpha, controls inducible transcription of the proinflammatory cytokine IL-1alpha. J Immunol. 2015:195(4):1359-63.

19. Cope AP, Feldmann M. Emerging approaches for the therapy of autoimmune and chronic inflammatory disease. Curr Opin Immunol. 2004;16(6):780-6.

20. Chatenoud L. Immune therapies of autoimmune diseases: are we approaching a real cure? Curr Opin Immunol. 2006;18(6):710-7.

21. Borchers AT, Leibushor N, Naguwa SM, Cheema GS, Shoenfeld Y, Gershwin ME. Lupus nephritis: a critical review. Autoimmun Rev. 2012;12(2):174-94.

22. Suarez-Gestal M, Calaza M, Endreffy E, Pullmann R, Ordi-Ros J, Sebastiani GD, et al. Replication of recently identified systemic lupus erythematosus genetic associations: a case-control study. Arthritis Res Ther. 2009;11(3):R69.

23. Yamanaka $\mathrm{H}$. TNF as a target of inflammation in rheumatoid arthritis. Endocr Metab Immune Disord Drug Targets. 2015;15(2):129-34.

24. Gomez JA, Wapinski OL, Yang YW, Bureau JF, Gopinath S, Monack DM, et al. The NeST long ncRNA controls microbial susceptibility and epigenetic activation of the interferon-gamma locus. Cell. 2013;152(4):743-54.

25. Shi H, Yu CQ, Xie LS, Wang ZJ, Zhang P, Zheng LY. Activation of TLR9dependent p38MAPK pathway in the pathogenesis of primary Sjogren's syndrome in NOD/Ltj mouse. J Oral Pathol Med. 2014;43(10):785-91.

26. Barone F, Bombardieri M, Manzo A, Blades MC, Morgan PR, Challacombe SJ, et al. Association of CXCL13 and CCL21 expression with the progressive organization of lymphoid-like structures in Sjogren's syndrome. Arthritis Rheum. 2005;52(6):1773-84.

27. Horvath S, Nazmul-Hossain AN, Pollard RP, Kroese FG, Vissink A, Kallenberg CG, et al. Systems analysis of primary Sjogren's syndrome pathogenesis in salivary glands identifies shared pathways in human and a mouse model. Arthritis Res Ther. 2012;14(6):R238.

28. Greenwell-Wild T, Moutsopoulos NM, Gliozzi M, Kapsogeorgou E, Rangel Z, Munson PJ, et al. Chitinases in the salivary glands and circulation of patients with Sjogren's syndrome: macrophage harbingers of disease severity. Arthritis Rheum. 2011;63(10):3103-15.

29. Hu S, Wang J, Meijer J, leong S, Xie Y, Yu T, et al. Salivary proteomic and genomic biomarkers for primary Sjogren's syndrome. Arthritis Rheum. 2007:56(11):3588-600.

30. Lisi S, Sisto M, Lofrumento DD, D'Amore M. Sjogren's syndrome autoantibodies provoke changes in gene expression profiles of inflammatory cytokines triggering a pathway involving TACE/NF-kappaB. Lab Invest. 2012;92(4):615-24.

31. Kino T, Hurt DE, Ichijo T, Nader N, Chrousos GP. Noncoding RNA gas5 is a growth arrest- and starvation-associated repressor of the glucocorticoid receptor. Sci Signal. 2010;3(107):ra8.

32. Haywood ME, Rose SJ, Horswell S, Lees MJ, Fu G, Walport MJ, et al. Overlapping BXSB congenic intervals, in combination with microarray gene expression, reveal novel lupus candidate genes. Genes Immun. 2006;7(3):250-63.

33. Koenders Ml, van den Berg WB. Novel therapeutic targets in rheumatoid arthritis. Trends Pharmacol Sci. 2015;36(4):189-95.

34. Hakonarson H, Grant SF, Bradfield JP, Marchand L, Kim CE, Glessner JT, et al. A genome-wide association study identifies KIAA0350 as a type 1 diabetes gene. Nature. 2007;448(7153):591-4.

35. Wallace C, Smyth DJ, Maisuria-Armer M, Walker NM, Todd JA, Clayton DG. The imprinted DLK1-MEG3 gene region on chromosome 14q32.2 alters susceptibility to type 1 diabetes. Nat Genet. 2010;42(1):68-71. 
36. Burfoot RK, Jensen CJ, Field J, Stankovich J, Varney MD, Johnson LJ, et al. SNP mapping and candidate gene sequencing in the class I region of the HLA complex: searching for multiple sclerosis susceptibility genes in Tasmanians. Tissue Antigens. 2008;71(1):42-50.

37. Sandhya P, Joshi K, Scaria V. Long noncoding RNAs could be potential key players in the pathophysiology of Sjogren's syndrome. Int J Rheum Dis. 2015;18:898-905.

38. Jonsson R, Klareskog L, Backman K, Tarkowski A. Expression of HLA-D-locus (DP, DQ, DR)-coded antigens, beta 2-microglobulin, and the interleukin 2 receptor in Sjogren's syndrome. Clin Immunol Immunopathol. 1987;45(2):235-43.

39. Manoussakis MN, Dimitriou ID, Kapsogeorgou EK, Xanthou G, Paikos S, Polihronis $\mathrm{M}$, et al. Expression of B7 costimulatory molecules by salivary gland epithelial cells in patients with Sjogren's syndrome. Arthritis Rheum. 1999;42(2):229-39.

40. Tsunawaki S, Nakamura S, Ohyama Y, Sasaki M, Ikebe-Hiroki A, Hiraki A, et al. Possible function of salivary gland epithelial cells as nonprofessional antigen-presenting cells in the development of Sjogren's syndrome. J Rheumatol. 2002;29(9):1884-96.

41. Pertovaara M, Korpela M. ESSPRI and other patient-reported indices in patients with primary Sjogren's syndrome during 100 consecutive outpatient visits at one rheumatological clinic. Rheumatology (Oxford). 2014;53(5):927-31.

42. Gottenberg JE, Seror R, Miceli-Richard C, Benessiano J, Devauchelle-Pensec V, Dieude $P$, et al. Serum levels of beta2-microglobulin and free light chains of immunoglobulins are associated with systemic disease activity in primary Sjogren's syndrome. Data at enrollment in the prospective ASSESS cohort. PloS one. 2013:8(5):e59868.

43. Ulitsky I, Bartel DP. lincRNAs: genomics, evolution, and mechanisms. Cell. 2013;154(1):26-46.

\section{Submit your next manuscript to BioMed Central and we will help you at every step:}

- We accept pre-submission inquiries

- Our selector tool helps you to find the most relevant journal

- We provide round the clock customer support

- Convenient online submission

- Thorough peer review

- Inclusion in PubMed and all major indexing services

- Maximum visibility for your research

Submit your manuscript at www.biomedcentral.com/submit 